\title{
UV abundances in HE0107-5240
}

\author{
Michael S. Bessell ${ }^{1}$ and Norbert Christlieb ${ }^{2}$ \\ ${ }^{1}$ RSAA, The Australian National University (Australia) \\ email: bessel@mso.anu.edu.au \\ ${ }^{2}$ Hamburger Sternwarte, Universitat Hamburg (Germany)
}

\begin{abstract}
We have determined the $\mathrm{CNO}$ abundances from $\mathrm{OH}, \mathrm{CH}$ and $\mathrm{NH}$ lines in the VLT/UVES UV spectra of HE0107-5240 and CD-38 245 together with the abundances of several metal species, including FeII, whose lines were not detected in blue spectra. The UV abundance analyses support those determined from the blue spectra, in particular the abundance from the FeII lines is in good agreement with the Fe abundance derived from the Fe I lines. It seems unlikely that the large overabundances of CNO have been produced in a medium-mass AGB star which now evolved to a white dwarf. The oxygen abundance is consistent with the predictions of Umeda \& Nomoto $(2003,2005)$, who proposed that HE0107-5240 has formed from a gas cloud which has been enriched by the yields of $\mathrm{a} \approx 25$ solar mass Population III star exploding as a supernova of low explosion energy $\mathrm{E}=3.10^{50} \mathrm{erg}$ with mixing and fallback. The odd-even effect predicted in the original models are not evident in the Sc and Co abundances of HE0107-5240, which are better fitted by the low density variant progenitor model.
\end{abstract}

Keywords. Astrochemistry, abundances, techniques: spectroscopic

\section{Introduction}

HE0107-5240 has been found by Christlieb et al. $(2002,2003)$ to be a giant with an iron abundance of $[\mathrm{Fe} / \mathrm{H}] \mathrm{NLTE}=-5.3$ and large overabundances of carbon and nitrogen $([\mathrm{C} / \mathrm{Fe}]=+4.0$ dex; $[\mathrm{N} / \mathrm{Fe}]=+2.3)$. Additional UVES spectra of HE0107-5240 and CD38245 covering the UV part of the spectrum below $3870 \mathrm{~A}$ were obtained, primarily to enable the oxygen abundance to be derived from the $\mathrm{OH}$ lines. The high oxygen overabundance of $\mathrm{HE} 0107-5240$ of $[\mathrm{O} / \mathrm{Fe}]=+2.3$ derived from these spectra was recently published by Bessell,Christlieb \& Gustafsson (2004). In this paper we report the UV analyses of $\mathrm{CH}, \mathrm{NH}$ and the metal lines of other species, such as Sc II, Co I and Fe II that were not seen in the blue spectra.

\section{Discussion}

\subsection{The Line lists}

Data for atomic and molecular lines for the spectrum synthesis and fine programs were taken from several sources. The VALD-2 (Kupka et al. 1999) database was used for the neutral and ionized atomic species. For preference, LIFBASE (Luque \& Crosley 1999) was used for the molecular lines as it based on the most recent and reliable molecular constants. LIFBASE was used to generate the wavelengths and transition probabilities for the various lines associated with different branches for the $\mathrm{C}-\mathrm{X} \mathrm{OH}$ and $\mathrm{CH}$ bands; but the corresponding upper and lower energy levels for each line were obtained by crosscorrelating the wavelengths, branches and $\mathrm{J}$ values of the transitions with those in the Kurucz (Kurucz, 1994) molecular database. For J values higher than provided by Kurucz, energy levels were extrapolated. The Kurucz linelist was used for $\mathrm{NH}$ but the gf values 
Table 1. UV Abundance Analyses

\begin{tabular}{lccccccc}
\hline & \multicolumn{4}{c}{ HE0107-5240 } & \multicolumn{3}{c}{ CD-38 245 } \\
\hline Species & Solar & Rel Sun & sd & No & Rel Sun & sd & No \\
\hline Mg I & -4.42 & -5.12 & .07 & 1 & -3.79 & .07 & 4 \\
Ca II & -5.64 & & & & -4.34 & .20 & 3 \\
Sc II & -8.91 & -5.54 & .25 & 2 & -3.96 & .08 & 13 \\
Ti I & -7.01 & & & & -3.78 & .08 & 5 \\
Ti II & -7.01 & -5.45 & .22 & 26 & -3.82 & .25 & 99 \\
V II & -8.00 & & & & -4.03 & .09 & 9 \\
Cr II & -6.33 & & & & -4.36 & .12 & 9 \\
Mn II & -6.61 & & & & -4.62 & .28 & 3 \\
Fe I & -4.49 & -5.42 & .11 & 49 & -4.24 & .20 & 110 \\
Fe II & -4.49 & -5.63 & .20 & 4 & -4.01 & .13 & 11 \\
Co I & -7.09 & -4.70 & .47 & 2 & -3.50 & .16 & 41 \\
\hline
\end{tabular}

were decreased by 0.63 dex to fit the NH lines in the sun. This then produced gf values 0.3 dex less than the values used by Carbon et al. (1982).

\subsection{The Carbon and Nitrogen Abundance}

The carbon abundance in HE0107-5240 was derived by fitting the higher order transitions away from the band head; however, the deep band head could not be fitted indicating that the model is probably insufficiently cool in the upper layers. Synthesis fits to the $\mathrm{CH}$ lines in HE0107-5240 away from the bandhead yielded $[\mathrm{C} / \mathrm{Fe}]=+3.6$. In CD-38 245 the $\mathrm{CH}$ bandhead was extremely weak and it yielded a $\mathrm{C}$ deficiency $[\mathrm{C} / \mathrm{Fe}]=-1.0$. Unlike $\mathrm{CH}$ and $\mathrm{OH}$, the UV NH lines are closely confined around the bandhead at 3160A. In HE01075240 the NH feature is very weak but clearly present indicating a nitrogen abundance of $[\mathrm{N} / \mathrm{Fe}]=+2.2$. In $\mathrm{CD}-38245 \mathrm{NH}$ was very strong and a nitrogen overabundance of $[\mathrm{N} / \mathrm{Fe}]=+1.3$ was fitted.

\subsection{The UV Metal Lines}

In CD-38 245 there were many lines of FeI, FeII, NiI, CoI and several lines of CrII, VII, ScII, MgI, CaII but in HE0107-5240 with its much lower metal abundance only a few of the strongest lines were visible. Nevertheless, several FeII lines were measured that enabled a check to be made on the ionization equilibrium and the effective gravity. Good constraints were also able to be placed on the $[\mathrm{Co} / \mathrm{Fe}]$ and $[\mathrm{Sc} / \mathrm{Fe}]$ abundances that are relevant to the predicted abundance pattern of the Umeda \& Nomoto $(2003,2005)$ supernovae fall-back models and support a lower density progenitor model and/or yields modified by invoking bipolar explosive jets.

\section{References}

Bessell, M.S., Christlieb, N. \& Gustafsson, B. 2004, ApJ, 612, L61

Carbon, D. F., Romanishin, W. et al. 1982 ApJS, 49, 207

Christlieb, N., Bessell, M., Beers, T. et al. 2002, Nature, 419, 904

Christlieb, N., Gustafsson, B., Korn, A., et al. 2003, ApJ, 603, 708

Kupka F., Piskunov, N.et al. A\&AS 138, 119 (1999) (VALD-2)

Kurucz, R. 1994 CD-ROM 1-23, Smithsonian Astrophysical Observatory.

Luque \& Crosley 1999; http://www.sri.com/cem/lifbase/Lifbase.PDF

Umeda, H. \& Nomoto, K. 2003, Nature, 422, 871

Umeda, H. \& Nomoto, K. 2005 ApJ 619, 427 